\title{
A RENORMING THEOREM FOR DUAL SPACES
}

\author{
A. C. YORKE \\ (Received 10 November 1979, revised 10 July 1982) \\ Communicated by E. Strzelecki
}

\begin{abstract}
If the second dual of a Banach space $E$ is smooth at each point of a certain norm dense subset, then its first dual admits a long sequence of norm one projections, and these projections have ranges which are suitable for a transfinite induction argument. This leads to the construction of an equivalent locally uniformly rotund norm and a Markuschevich basis for $E^{*}$.
\end{abstract}

1980 Mathematics subject classification (Amer. Math. Soc.): 46 B 99.

\section{Preliminaries}

Let $E$ be a real Banach space, $E^{*}$ its dual, $\hat{E}$ the canonical embedding of $E$ in $E^{* *}$, and $S(E)$ the unit sphere of $E$. The mapping $D: E \rightarrow 2^{E^{*}}$ which associates with each $x \in E$ the $\left\{f \in E^{*}: f(x)=\|f\|\|x\|\right.$ and $\left.\|f\|=\|x\|\right\}$ is called the duality mapping. The duality mappings on $E^{*}, E^{* *}$, and $Y$, a subspace of $E$, will be denoted by $D_{1}, D_{2}$, and $D_{Y}$, respectively. The set $D(S(E))$ will be denoted by $D(S)$ and elements of $D(S)$ by $f_{x}$. Recall that the Bishop-Phelps Theorem states that $D(S)$ is norm dense in $S\left(E^{*}\right)$.

$E$ is smooth [very smooth] at $x \in S(E)$ if $D(x)\left[D_{2}(\hat{x})\right]$ is a singleton (see Giles (1975) and Yorke (1977) for equivalent definitions). $E$ is smooth [very smooth] if $E$ is smooth [very smooth] at each $x \in S(E) . E$ is rotund if $x, y \in S(E)$ with $\|x+y\|=2$ implies $x=y$. $E$ is weakly locally uniformly rotund (WLUR) [locally uniformly rotund (LUR)] at $x \in S(E)$ if every sequence (or net) $\left\{x_{n}\right\}$ in $S(E)$ with $\left\|x_{n}+x\right\| \rightarrow 2$ has $x_{n} \rightarrow x$ in the $\sigma\left(E, E^{*}\right)$ topology [the norm topology]. $E$ is $W L U R(L U R)$ if $E$ is WLUR [LUR] at each $x \in S(E)$. 
A biorthogonal set $\left\{\left(x_{i}, f_{i}\right): i \in I\right\} \subset E \times E^{*}$ is called a Markuschevich basis (M-basis) for $E$ if $E=\overline{\operatorname{sp}}\left\{x_{i}\right\}$ and $\overline{\operatorname{sp}}\left\{f_{i}\right\}$ is total over $E$. The density character of a subspace $Y \subset E$, denoted dens $Y$, is the minimum cardinality of a (relatively) norm dense subset of $Y$. E is weakly compactly generated $(W C G)$ if there is a

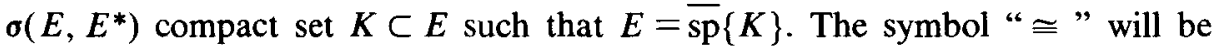
used to mean "isometrically isomorphic to".

Tacon (1970) showed that if $E^{* *}$ is smooth at each point of $S(\hat{E})$, that is, if $E$ is very smooth, then $E^{*}$ can be equivalently renormed to be rotund. The main step in obtaining this norm involves the construction of a long sequence $\left\{P_{\alpha}\right.$ : $\left.\omega \leqslant \alpha \leqslant \mu, \overline{\bar{\mu}}=\operatorname{dens} E^{*}\right\}$ of norm one projections on $E^{*}$. However, in order to use the transfinite induction method of Troyanski (1971) to improve this result, one must be able to identify each $\left(P_{\alpha+1}-P_{\alpha}\right) E^{*}, \omega \leqslant \alpha<\mu$, with the dual of a very smooth space, or, equivalently, prove that certain quotient spaces of $E$ are also very smooth. The purpose of this paper is to show that if $E^{* *}$ is given a stronger type of partial smoothness: if $E^{* *}$ is smooth at each $F_{f} \in D_{1}(S)$, then the necessary amount of smoothness will pass to the required quotient spaces. Now the standard transfinite induction argument can be used to construct an $M$-basis and an equivalent LUR norm for $E^{*}$. Unfortunately, as with Tacon's rotund norm, this LUR norm need not be a dual norm.

\section{The theorem}

In what follows the expression " $D(S)$ is smooth" will be used to mean that $E^{*}$ is smooth at each $f_{x} \in D(S)$.

Proposition. Let $Y$ be a subspace of $E$. If $D(S)$ is smooth, then so is $D_{Y}(S)$.

Proof. If $Y^{*}$ is not smooth at $f \in D_{Y}(S)$ there are sequences $\left\{y_{n}\right\}$ and $\left\{z_{n}\right\}$ in $S(Y)$, a $g \in S\left(Y^{*}\right)$, and an $\varepsilon>0$ such that $f\left(y_{n}+z_{n}\right) \rightarrow 2$ but $\left|g\left(y_{n}-z_{n}\right)\right| \geqslant \varepsilon$ for all $n$ (Yorke (1977; Proposition 3)). But, by the Hahn-Banach Theorem, there are $\tilde{f}$ and $\tilde{g}$ in $S\left(E^{*}\right)$ such that $\tilde{f}=f$ and $\tilde{g}=g$ on $Y$. Thus $\left|\tilde{g}\left(y_{n}-z_{n}\right)\right| \geqslant \varepsilon$ for all $n$ even though $\tilde{f}\left(y_{n}+z_{n}\right) \rightarrow 2$, hence $E^{*}$ is not smooth at $\tilde{f}$. However, since $f \in D_{Y}(S)$ and $\tilde{f}=f$ on $Y, f \in D(S)$, so $D(S)$ can not be smooth either.

Lemma. Let $D_{1}(S)$ be smooth. Then there is a transfinite sequence of projections $\left\{T_{\delta}: \delta \in D\right\}$ defined on $E^{*}$ such that, for each $\delta$,

(1) $\left\|T_{\delta}\right\|<\infty$,

(2) $T_{\delta} T_{\gamma}=T_{\gamma} T_{\delta}=T_{\delta}$ if $\gamma<\delta$,

(3) $\cup_{\gamma<\delta} T_{\gamma+1} E^{*}$ is norm dense in $T_{\delta} E^{*}$, 
(4) for any $\varepsilon>0$ and $f \in E^{*}$ the $\left\{d:\left\|\left(T_{\delta+1}-T_{\delta}\right) f\right\|>\varepsilon\left(\left\|T_{\delta}\right\|+\left\|T_{\delta+1}\right\|\right)\right\}$ is finite,

(5) $\left(T_{\delta+1}-T_{\delta}\right) E^{*}$ is separable, and

(6) $\overline{\bigcup_{\delta \in D}\left(T_{\delta+1}-T_{\delta}\right) E^{*}}=E^{*}$.

Proof. Let $\mu$ be the first ordinal number of cardinality dens $E^{*}$. Then since $D_{1}(S)$ is smooth and $S(\hat{E}) \subset D_{1}(S), E$ is very smooth. Thus for every $\alpha, \omega \leqslant \alpha \leqslant$ $\mu$, there is a subspace $E_{\alpha} \subset E$, with dens $E_{\alpha} \leqslant \overline{\bar{\alpha}}$, and a projection $P_{\alpha}$ on $E^{*}$ such that

(1) $\left\|P_{\alpha}\right\|=1$,

(2) $P_{\alpha} E^{*}=D \overline{\left(E_{\alpha}\right)} \cong E_{\alpha}^{*}$,

(3) $P_{\alpha} P_{\beta}=P_{\beta} P_{\alpha}=P_{\beta}$ if $\beta<\alpha$,

(4) $\cup_{\beta<\alpha} P_{\beta+1} E^{*}$ is norm dense in $P_{\alpha} E^{*}$,

(5) $P_{\mu}=I$, and

(6) $\left(P_{\alpha+1}-P_{\alpha}\right) E^{*} \cong\left(E_{\alpha+1} / E_{\alpha}\right)^{*}, \alpha<\mu$.

(For (1) through (5) use Tacon (1970; Theorem 2); (6) is given in John and Zizler (1975, Lemma 1(8)).) Now proceed by induction on dens $E^{*}$. If $E^{*}$ is separable the result is immediate. Assume that the lemma holds for all cardinal numbers less than dens $E^{*}$. Let $G_{\alpha}$ denote $E_{\alpha+1} / E_{\alpha}$ for each $\alpha, \omega \leqslant \alpha<\mu$. Since $G_{\alpha}^{*} \cong$ $\left(P_{\alpha+1}-P_{\alpha}\right) E_{\alpha}^{*}$ and $D_{1}(S)$ is smooth, the proposition shows that each $D_{G_{\alpha}^{*}}(S)$ is smooth. (This means that each $E_{\alpha+1} / E_{\alpha}$ is very smooth.) Thus, since dens $\left(P_{\alpha+1}-P_{\alpha}\right) E^{*}<\overline{\bar{\mu}}, \omega \leqslant \alpha<\mu$, the inductive hypothesis gives a transfinite sequence of projections $\left\{S_{\beta}^{\alpha}: \omega \leqslant \beta \leqslant \Gamma_{\alpha}, \overline{\bar{\Gamma}}_{\alpha}=\operatorname{dens}\left(P_{\alpha+1}-P_{\alpha}\right) E^{*}\right\}$ on each $\left(P_{\alpha+1}-P_{\alpha}\right) E^{*}$ which satisfies the conditions of the lemma. Let $D=\{(\alpha, \beta)$ : $\omega \leqslant \alpha \leqslant \mu, \omega \leqslant \beta \leqslant \Gamma_{\alpha}$ \} and order this set lexiographically. For each $\delta \in D$ define

$$
T_{\delta}= \begin{cases}S_{\alpha}^{\beta}\left(P_{\alpha+1}-P_{\alpha}\right)+P_{\alpha} & \text { if } \alpha<\mu\left(S_{\omega}^{\omega}=0\right), \\ I & \text { if } \alpha=\mu .\end{cases}
$$

Now as in Troyanski (1971, page 177) one can show that the elements of $\left\{T_{\delta}\right.$ : $\delta \in D\}$ have the desired properties.

THEOREM. If $D_{1}(S)$ is smooth, then

(1) $E^{*}$ admits an $M$-basis, and

(2) $E^{*}$ can be equivalently renormed to be LUR.

Proof. (1) Use the standard transfinite induction argument (for example, see John and Zizler (1975, page 294)).

(2) Since $D_{1}(S)$ is smooth, $E$ is very smooth, hence there is a continuous one-to-one linear operator which maps $E^{*}$ into $c_{0}(\Gamma)$ for some set $\Gamma$ (Tacon 
(1970, Theorem 1)): This, together with properties (3), (4), (5), and (6) of the lemma, shows that $E^{*}$ satisfies the conditions of Proposition 1 of Troyanski (1971, page 175).

COROllary. If $E^{*}$ is WLUR, then

(1) $E^{*}$ admits an $M$-basis, and

(2) $E^{*}$ can be equivalently renormed to be LUR.

Proof. $E^{*}$ WLUR implies $D_{l}(S)$ is smooth (Yorke (1979, Theorem 1)).

Notice that although this WLUR norm for $E^{*}$ must be a dual norm, the LUR norm, although equivalent, need not be dual.

If the projections of the lemma are $\sigma\left(E^{*}, E\right)$ continuous it is not difficult to show that $E$ must be WCG. On the other hand if $E$ is WCG, as well as very smooth, then the projections will be $\sigma\left(E^{*}, E\right)$ continuous (use John and Zizler (1974, Lemma 4)). However, even though it is likely that if $E^{* *}$ is smooth, $E$ is WCG, it appears that the methods used in the proof of the lemma will not give this result without additional assumptions.

\section{References}

D. Amir and J. Lindenstrauss (1968), 'The structure of weakly compact sets in Banach spaces,' Ann. of Math. (2) 88, 35-46.

J. Diestel (1975), Geometry of Banach spaces-selected topics (Springer-Verlag, Berlin, Heidelberg, New York).

J. R. Giles (1975), 'On smoothness of the Banach space embedding,' Bull. Austral. Math. Soc. 13, 69-74.

K. John and V. Zizler (1974), 'Smoothness and its equivalents in weakly compactly generated Banach spaces', J. Functional Analysis 15, 1-11.

K. John and V. Zizler (1975), 'Markuschevich bases in some dual spaces,' Proc. Amer. Math. Soc. 50, 293-296.

D. G. Tacon (1970), 'The conjugate of a smooth Banach space,' Bull. Austral. Math. Soc. 2, 415-425.

S. L. Troyanski (1971), 'On locally uniformly convex and differentiable norms in certain non-separable Banach spaces,' Studia Math. 37, 173-180.

A. C. Yorke (1977), 'Weakly rotundity in Banach spaces,' J. A ustral. Math. Soc. Ser. A 24, 224-233.

A. C. Yorke (1979), 'Differentiability and local rotundity,' J. Austral. Math. Soc. Ser. A 28, 205-213.

School of Mathematical and Physical Sciences

Murdoch University

Murdoch

Western Australia 6150 